\title{
Prompt Photon Production in $p-A$ Collisions at LHC and the Extraction of Gluon Shadowing
}

\author{
François Arleo $^{1 *}$ and Thierry Gousset ${ }^{2}$ \\ 1- CERN, PH Department, TH Division \\ 1211 Geneva 23, Switzerland \\ 2- SubaTech, Université de Nantes, Ecole des Mines de Nantes, IN2P3/CNRS \\ 4 rue Kastler, 44307 Nantes, France
}

\begin{abstract}
A report is given on the study of using prompt photon production at the LHC to probe the gluon nuclear density, and more specifically the shadowing ratio $G_{A} / G_{p}$ that one could access in foreseen $p-\mathrm{A}$ runs.
\end{abstract}

\section{Motivations}

In the framework of collinear factorization in QCD, shadowing corrections show up as leading-twist modifications of per-nucleon parton densities

$$
u_{p}\left(x, Q^{2}\right) \rightarrow u_{A}\left(x, Q^{2}\right), G_{p}\left(x, Q^{2}\right) \rightarrow G_{A}\left(x, Q^{2}\right), \ldots
$$

that can also be described as deviations from 1 of ratios such as

$$
R_{G}^{(A)}\left(x, Q^{2}\right)=G_{A}\left(x, Q^{2}\right) / G_{p}\left(x, Q^{2}\right) .
$$

These deviations are worth knowing in the shadowing region, i.e. $x<10^{-1}$ for at least two reasons. First, collinear factorization is used to estimate cross sections for hard processes in $\mathrm{A}-\mathrm{A}$ that are used as baselines from which observed deviations may signal genuine medium effects. Second, it is also interesting in itself for the understanding of the high-parton density regime in QCD.

Nuclear parton densities are extracted from deep inelastic scattering and Drell-Yan data [2]. Several global fits, including DGLAP evolution, exist (EKS [3], HKM [4], nDS [5]) as well as model calculations [6]. The recent $N L O$ analysis from de Florian and Sassot [5] is used in the following.

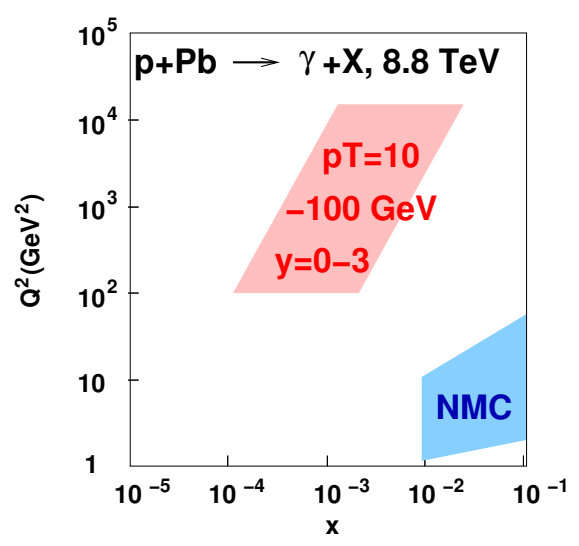
One important point which motivates the present analysis is the practically unconstrained amount of glue in a nucleus.

\footnotetext{
* On leave from Laboratoire d'Annecy-le-Vieux de Physique Théorique (LAPTH), UMR 5108 du CNRS ass ociée à l'Université de Savoie, B.P. 110, 74941 Annecy-le-Vieux Cedex, France
} 


\section{Inclusive photons}

Prompt photons are produced at the parton level and do not result from the decay of a produced hadron such as a $\pi^{0}$. Prompt photon production at large $p_{T}$ has a cross section

$$
\begin{aligned}
d \sigma(p+p \rightarrow \gamma+X) \stackrel{\text { LO }}{=} & u_{1} * \bar{u}_{2} * d \hat{\sigma}(u+\bar{u} \rightarrow \gamma+g)+ \\
& u_{1} * G_{2} * d \hat{\sigma}(u+g \rightarrow \gamma+u)+\cdots+ \\
& u_{1} * G_{2} * D_{u}^{\gamma} * d \hat{\sigma}(u+g \rightarrow u+g)+\cdots
\end{aligned}
$$

On the right-hand side, the first two lines are examples of direct production, whereas the third line shows a fragmentation channel. In the kinematical region discussed below, direct production and fragmentation are of comparable importance. For direct production, the Compton channel (second line) is one order of magnitude stronger than the annihilation one (first line).

The cross section

$$
\frac{d^{3} \sigma}{d y d^{2} p_{T}}(p+p \rightarrow \gamma+X)
$$

can be studied as a function of $s, p_{T}$, and $y$. It has been measured at several energies, with various projectiles, giving a rather rich phenomenology. Collider data are fairly well described by pQCD at NLO [7].

The ratio

$$
R_{p A}=\frac{d \sigma(p+A \rightarrow \gamma+X)}{A d \sigma(p+p \rightarrow \gamma+X)}
$$

is studied at $\sqrt{s}=8.8 \mathrm{TeV}$ as a function of $x_{T}=p_{T} /(\sqrt{s} / 2)$ and $y$ with INCNLO [8], assuming either proton or nuclear parton densities. The result is shown in Fig. 2 .
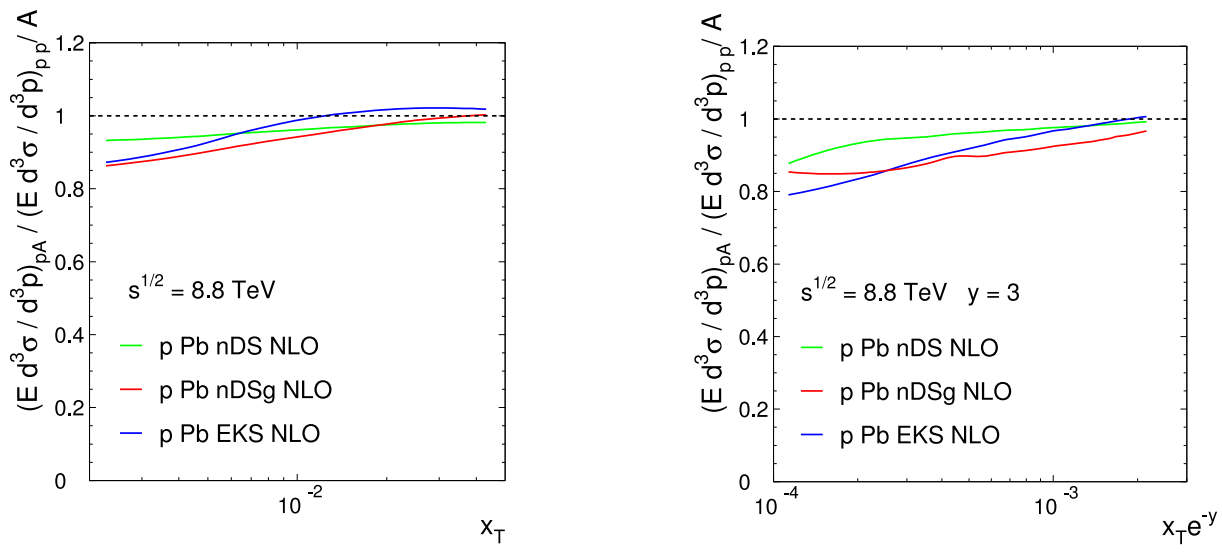

Figure 2: Ratio of inclusive photons at $y=0$ and $y=3$ in $p-\mathrm{Pb}$ over $p-p$ collisions at $\sqrt{s}=8.8 \mathrm{TeV}$.

The shadowing ratio, $R_{p A}$ is plotted vs $x_{T} e^{-y}$ that is indicative of the $x_{2}$ region probed in the target. The process is sensitive to modifications of parton densities and to the change of isospin composition. 


\section{$3 \quad$ Isolated photons}

In order to cut out the $\pi^{0}$ background, it is often useful to select prompt photon candidates by way of an isolation criterion. This also eliminates to a large extent the fragmentation component. As a consequence, a direct extraction of shadowing ratios can be devised.

For the direct channel the cross section writes $d \sigma=f_{1} * f_{2} * d \hat{\sigma}$. The $x$ region probed is selected by the behavior of the parton densities. Ratios such as $R_{G}=G_{A} / G_{p}$ show much less variation. Therefore an approximate expression of $d \sigma_{p A}$ can be obtained by factorizing the parton density ratio out of the convolution. At which $x$ this factorization takes place? To answer this, a closer look to the convolution is necessary.

At leading order, the Compton cross section is

$$
\begin{aligned}
\frac{d^{3} \sigma}{d y d^{2} p_{T}} \propto \int d v \quad & F^{(1)}\left(\frac{x_{T} e^{y}}{2 v}\right) G^{(2)}\left(\frac{x_{T} e^{-y}}{2(1-v)}\right)\left(1-v+\frac{1}{1-v}\right) \\
& +G^{(1)}\left(\frac{x_{T} e^{y}}{2 v}\right) F^{(2)}\left(\frac{x_{T} e^{-y}}{2(1-v)}\right)\left(v+\frac{1}{v}\right),
\end{aligned}
$$

where $F^{(1)}(x)\left(F^{(2)}(x)\right)$ stands for the projectile (target) structure function $F_{2}(x)$ divided by $x$. At small $x_{T}$ (and not-too-large $|y|$ ), $F(x) \sim A x^{-a}$ and $G(x) \sim B x^{-b}$ which translates into $F \times G \propto v^{a}(1-v)^{b}$. Since $a$ and $b$ are positive and close to each other the change $R \rightarrow R\left(x_{T} e^{-y}\right)$ is suggested. At $y=0$, both terms in Eq. (1) contributes the same amount in $p-p$ and the nuclear production ratio is $\approx 0.5\left(R_{G}+R_{F_{2}}\right)$. At $y=3$, the second term in Eq. (1) is suppressed relative to the first and the nuclear production ratio is $\approx R_{G}$.

The nuclear ratio for isolated photons was computed with JETPHOX [7], using an isolation criterion: $E_{T}^{\mathrm{had}} / p_{T}^{\gamma} \leq 0.1$ in a cone of radius $R=0.4$ around the photon. The result is shown in Fig. 3 (open circles) and compared with $0.5\left(R_{G}+R_{F_{2}}\right)$ (dash-dotted line).

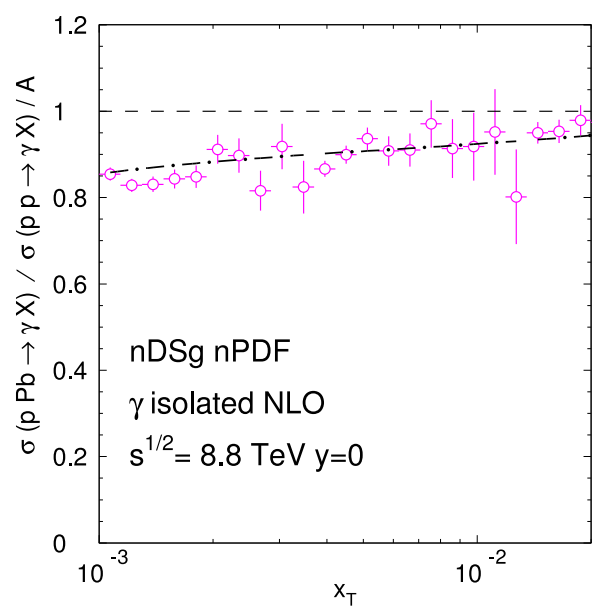

Figure 3: Ratio of isolated photons produced at $y=0$ in $p-\mathrm{Pb}$ over $p-p$ collisions at $\sqrt{s}=8.8 \mathrm{TeV}$. 


\section{Outlook}

Nuclear modifications up to $20 \%$ are expected from the extrapolation of the fits made to NMC data and used here to predict nuclear production ratios in prompt photon production at the LHC. This represents challenging measurements, especially because of systematic uncertainties that should be further investigated. It also requires the same energy for $p-p$ and $p-A$ or some extrapolation.

\section{References}

[1] Slides:

http://indico. cern. ch/contributionDisplay $\cdot$ py? contribId=50\&sessionId=8\& conf Id=9499

[2] M. Arneodo, Phys. Rep. 240301 (1994).

[3] K. Eskola, V. Kolhinen and C. Salgado, Eur. Phys. J. 961 (1999).

[4] M. Hirai, S. Kumano and M. Miyama, Phys. Rev. D64 034003 (2001).

[5] D. de Florian and R. Sassot, Phys. Rev. D69 074028 (2004).

[6] N. Armesto, J. Phys. G 32 R367 (2006).

[7] P. Aurenche et al, Phys. Rev. D73 094007 (2006).

[8] P. Aurenche et al, Eur. Phys. J. 9107 (1999). 